Check for updates

Cite this: RSC Adv., 2018, 8, 29756

\title{
Dewatering of drilling sludge by ultrasound assisted Fe(II)-activated persulfate oxidation
}

\begin{abstract}
Liyan Liu, (D) Hao Yan, Chao Yang and Guorui Zhu id †*
An ultrasound assisted Fe(॥)-activated persulfate oxidation method was put forward to improve the dewaterability of drilling sludge in this research. The water content in the filter cake and specific resistance to filtration (SRF) were measured to evaluate the sludge dewaterability. Volatile suspended solids (VSS), transmittance of supernatant, microstructure, particle size distribution and zeta potential were tested to justify the proposed mechanism. The results showed that appropriate ultrasound assisted Fe(II)-activated persulfate oxidation could not only further enhance the sludge dewaterability but also reduce the reaction time as well. The optimal conditions for this method were $1.6 \%$ sodium persulfate, $0.8 \%$ ferrous sulfate, $40 \mathrm{~W}$ ultrasonic power and $45 \mathrm{~min}$ reaction time. Reduction of VSS and an increase of transmittance were further achieved compared to oxidation alone. SEM results and the decrease of particle size after the treatment confirmed the disintegration of sludge flocs, which promoted the release of bound water. A synergistic effect mechanism of ultrasound and chemical oxidation was proposed, with ultrasonic cavitation disintegrating the flocs, exposing the interior organics and persulfate further oxidizing the released organics.
\end{abstract}

Received 19th April 2018

Accepted 15th August 2018

DOI: $10.1039 / \mathrm{c} 8 \mathrm{ra03376e}$

rsc.li/rsc-advances sludge dewaterability as ultrasound power increases. ${ }^{12}$ Acidification enhances the dewaterability of oily sludge by changing the floc structure. ${ }^{13}$ Advanced Oxidation Processes (AOPs), based on the generation of highly reactive chemical oxidants such as hydroxyl radicals in Fenton $\left(\mathrm{Fe}^{2+} / \mathrm{H}_{2} \mathrm{O}_{2}\right)$ process and sulfate radicals in $\mathrm{Fe}(\mathrm{II})-\mathrm{S}_{2} \mathrm{O}_{8}{ }^{2}$ oxidation process, degrade organics in the sludge from large molecular sizes into smaller sizes and oxidize them in a short time..$^{14,15}$ The mechanism of most of these approaches are disruption of the sludge flocs and solubilization of the exposed organics. With the disintegration of sludge floc structure, the release of bound and interstitial water trapped between flocs could be facilitated. ${ }^{16}$ In recent years, combination of different pretreatment methods have attracted much attention, ${ }^{17}$ among which ultrasonic treatment and advanced oxidation technology are considered as promising methods.

Ultrasonic cavitation can lead to high shear forces and extreme conditions in micro-regions with estimated temperature as high as $5000 \mathrm{~K}$ and pressure up to $1 \times 10^{8} \mathrm{~Pa}$, generating hydroxyl radicals and resulting in acceleration of chemical reactions and sludge disintegration..$^{18}$ Several reports have showed that ultrasonic pretreatment has both positive and negative influence on the sludge dewaterability. Na et al. ${ }^{19}$ demonstrated that the particle size of digested sludge after ultrasonic treatment decreases and its dewaterability is improved significantly. The capillary suction time reduces with the increase of supplied ultrasonic energy. Chu et al. ${ }^{20}$ observed that the sludge floc structure can be effectively disintegrated and organic substances can be released after ultrasonic
School of Chemical Engineering and Technology, Tianjin University, Tianjin 300350, China.E-mail: zhuguorui@tju.edu.cn; Fax:+862227408728; Tel: +862227408728

$\dagger$ Mail address: 135 Yaguan Rd, Haihe Educational Park, Tianjin 300350, China. 
pretreatment, but the sludge dewaterability markedly deteriorates and the water content increases with ultrasonic power. Feng et al. ${ }^{12}$ concluded that low-energy dosage can enhance sludge dewaterability in terms of capillary suction time and specific resistance to filtration, while high-energy dosage has opposite effect. The optimal energy dosage is $800 \mathrm{~kJ} \mathrm{~kg}^{-1}$ total solids during ultrasonic treatment.

Traditional AOPs, Fenton and Fenton-like technology, have been extensively investigated for dewatering of sludge. ${ }^{21-23}$ The Fenton oxidation is the reaction of $\mathrm{H}_{2} \mathrm{O}_{2}$ and ferrous iron under acidic conditions, generating hydroxyl radicals. ${ }^{24}$ However, the strong acidic condition limits the application of Fenton reagent. Subsequently, Fe(II)-activated persulfate oxidation is used to condition waste activated sludge, the mechanism of which is based on the generation of the sulfate radicals, ${ }^{20}$ which still have high oxidizing potential at neutral or alkaline environment.

$$
\mathrm{Fe}^{2+}+\mathrm{S}_{2} \mathrm{O}_{8}^{2-} \rightarrow \mathrm{Fe}^{3+}+\mathrm{SO}_{4}^{-\cdot}+\mathrm{SO}_{4}^{2-}
$$

Zhen et $a l .{ }^{25}$ reported that $\mathrm{Fe}^{2+} / \mathrm{S}_{2} \mathrm{O}_{8}{ }^{2-}$ oxidation is an effective method for sludge conditioning, and the sulfate radical oxidation destroys the particular functional groups, breaking the polymeric backbone. Shi et al. ${ }^{26}$ found that the simultaneous application of $\mathrm{Fe}^{2+}$ activated sodium persulfate and skeleton builder promote sludge dewatering, and the highest reduction efficiencies of specific resistance to filtration and capillary suction time are $88.5 \%$ and $91.5 \%$, respectively. $\mathrm{Fe}^{2+} /$ $\mathrm{S}_{2} \mathrm{O}_{8}{ }^{2-}$ oxidation under mild temperature has been demonstrated to be effective in improving the dewaterability of waste activated sludge. ${ }^{27}$

Despite the extensive work researchers have done up till now, the above-mentioned methods are mainly used for waste activated sludge or municipal sludge, treatment of drilling sludge has hardly been discussed. In the present work, the use of $\mathrm{Fe}^{2+}$ / $\mathrm{S}_{2} \mathrm{O}_{8}{ }^{2-}$ oxidation in combination with ultrasound for the treatment of drilling sludge has been done for the first time to the best of our knowledge. The effects of sodium persulfate and ferrous sulfate concentration were studied to obtain the optimal conditions for sulfate radical oxidation. After that, the impact of ultrasound was studied under the optimal operating conditions. The water content and specific resistance to filtration (SRF) were measured to evaluate the sludge dewaterability. Besides, volatile suspended solids (VSS) and transmittance of the supernatant were tested to characterize the change of organic matter in drilling sludge. Furthermore, microscopic structure of sludge, zeta potential, and particle size distribution were tested to analyze the physicochemical properties of floc and address the possible mechanisms of drilling sludge dewatering.

\section{Materials and methods}

\subsection{Materials}

The drilling sludge was obtained from an oil field located in northeastern China. It has dark brown color and poor
Table 1 Basic characteristics of drilling sludge

\begin{tabular}{lr}
\hline Parameter & \multicolumn{1}{c}{ Value } \\
\hline Water content before filtration (\%) & $77.93 \pm 0.21$ \\
pH & $7.74 \pm 0.09$ \\
Viscosity $\left(\mathrm{mPa} \mathrm{s}^{\circ}\right)$ & $115.07 \pm 2.14$ \\
Density $\left(\mathrm{g} \mathrm{mL} \mathrm{mL}^{-1}\right)$ & $1.19 \pm 0.07$ \\
Solid density $\left.(\mathrm{g} \mathrm{mL})^{-1}\right)$ & $1.98 \pm 0.11$ \\
Volatile suspended solids $\left(\mathrm{mg} \mathrm{L}^{-1}\right)$ & $9980 \pm 107$ \\
Zeta potential $(\mathrm{mV})$ & $-23.2 \pm 0.3$
\end{tabular}

settleability. The basic characteristics of the sludge were analyzed in Table 1.

Sodium persulfate and ferrous sulfate were analytical reagents, which were purchased from Yuanli Chemical Reagents Co. (Tianjin, China). The chemical reagents were used as received without further purification.

\subsection{Orthogonal experiment}

The optimal conditions were determined by the orthogonal experiments with the value of water content of filter cake as the target index. Sodium persulfate concentration, ferrous sulfate concentration (the units of the chemical dosage are calculated based on the weight of the dried sludge, for example, $0.8 \% \mathrm{~g} \mathrm{~g}^{-1}$ sodium persulfate means the weight of the added sodium persulfate equaled to $0.8 \%$ of the dried sludge), stirring speed and reaction time with three levels were chosen in the orthogonal experiments (see Table 2).

The order of the influence on SRF, according to $R$-value, was: sodium persulfate concentration $>$ ferrous sulfate concentration $>$ reaction time $>$ stirring speed. Furthermore, from the analysis of variance for orthogonal experiments in Table 3, the order of sum of the deviation square was the same as that of $R$ value: sodium persulfate concentration $>$ ferrous sulfate concentration $>$ reaction time $>$ stirring speed.

\subsection{Experimental procedure}

A series of tests, sulfate radical oxidation and ultrasound assisted sulfate radical oxidation, were conducted in the $150 \mathrm{~mL}$ beakers with $50 \mathrm{~mL}$ (the density of the sludge is $1.19 \mathrm{~g} \mathrm{~mL}^{-1}$, which means the weight was $59.5 \mathrm{~g}$ ) sludge sample. The effects of sodium persulfate and ferrous sulfate concentration, reaction time and ultrasonic power on dewaterability of drilling sludge were investigated. As acidic condition leads to serious corrosion of equipment, the study was conducted at its inherent pH. For sulfate radical oxidation treatment, the effect of sodium persulfate and ferrous sulfate concentrations were evaluated while the concentration of ferrous sulfate and sodium persulfate were kept at $0.8 \% \mathrm{~g} \mathrm{~g}^{-1}$ and $1.6 \% \mathrm{~g} \mathrm{~g}^{-1}$, respectively. A specified sodium persulfate was added into the sludge samples, and the reaction was initiated immediately after adding an appropriate amount of ferrous sulfate into the samples. Then the sludge sample was continuously agitated at $300 \mathrm{rpm}$ for $45 \mathrm{~min}$. After the treatment, the sludge sample dewaterability was measured. For ultrasound assisted sulfate radical oxidation treatment, a low frequency ultrasonic apparatus (KQ-100VDE, 
Table 2 Orthogonal experiment arrangement and results

\begin{tabular}{|c|c|c|c|c|c|}
\hline & $\begin{array}{l}\text { Sodium persulfate } \\
\text { concentration }\left(\% \mathrm{~g} \mathrm{~g}^{-1}\right)\end{array}$ & $\begin{array}{l}\text { Ferrous sulfate } \\
\text { concentration }\left(\% \mathrm{~g} \mathrm{~g}^{-1}\right)\end{array}$ & $\begin{array}{l}\text { Stirring speed } \\
(\mathrm{rpm})\end{array}$ & $\begin{array}{l}\text { Reaction time } \\
(\min )\end{array}$ & $\begin{array}{l}\text { Water content } \\
\text { of filter cake (\%) }\end{array}$ \\
\hline 1 & 0.8 & 0.8 & 150 & 15 & 34.34 \\
\hline 3 & 0.8 & 2.4 & 450 & 45 & 33.94 \\
\hline 4 & 1.6 & 0.8 & 300 & 45 & 30.90 \\
\hline 5 & 1.6 & 1.6 & 450 & 15 & 32.46 \\
\hline 8 & 2.4 & 1.6 & 150 & 45 & 32.12 \\
\hline 9 & 2.4 & 2.4 & 300 & 15 & 33.63 \\
\hline$K 1$ & 67.92 & 64.64 & 67.04 & 67.02 & \\
\hline$K 2$ & 64.85 & 65.56 & 65.33 & 66.18 & \\
\hline K3 & 64.99 & 67.58 & 65.41 & 64.56 & \\
\hline$R$ & 3.07 & 2.94 & 1.71 & 2.46 & \\
\hline
\end{tabular}

Shumei, China) was used, and the frequency was fixed at 20 $\mathrm{kHz}$. The impacts of the ultrasonic reaction time and power on sludge dewaterability were investigated as follows. Sludge samples were transferred to the ultrasonic apparatus, following which sodium persulfate and ferrous sulfate dosage of $1.6 \% \mathrm{~g}$ $\mathrm{g}^{-1}$ and $0.8 \% \mathrm{~g} \mathrm{~g} \mathrm{~g}^{-1}$ were respectively added to the sludge samples. Then ultrasonic irradiation exposed to the sludge samples immediately. At the same time, the sludge samples were continuously agitated at $300 \mathrm{rpm}$ for the desired reaction time. Similarly, sludge dewaterability was analyzed after the conditioning. All the tests were performed in triplicate, and the average values were reported in this paper. The average temperature of the drilling sludge during the US treatment varied between 25 and $40{ }^{\circ} \mathrm{C}$.

\subsection{Analytical methods}

The SRF and water content were chosen as the indicator of sludge dewaterability. ${ }^{28,29}$ The Buchner funnel method was used to measure the SRF. The filtration was performed under vacuum 0.1 MPa until no filtrate flowed out in $1 \mathrm{~min}$, thus the SRF was calculated by the following equation: ${ }^{30,31}$

$$
\mathrm{SRF}=\frac{2 P A^{2} b}{\mu w}
$$

where SRF is the specific resistance to filtration $\left(\mathrm{m} \mathrm{kg}^{-1}\right), P$ is the filtration pressure $\left(\mathrm{N} \mathrm{m}^{-2}\right), A$ is the filter area $\left(\mathrm{m}^{2}\right), b$ is the slope of the filtrate discharge curve $\left(t V^{-1}\right.$ versus $\left.V\right)\left(\mathrm{s} \mathrm{m}^{-6}\right), \mu$ is the filtrate viscosity $\left(\mathrm{N} \mathrm{s} \mathrm{m}^{-2}\right), w$ is the weight of cake solids per unit volume of filtrate $\left(\mathrm{kg} \mathrm{m}^{-3}\right)$.

When the filtration process finished, the filter cake was removed, weighed and dried at $105^{\circ} \mathrm{C}$ for $24 \mathrm{~h}$ to determine the water content, using the following equation: ${ }^{32,33}$

$$
\text { Water content } \%=\left(1-\frac{W_{2}}{W_{1}}\right) \times 100
$$

where $W_{1}$ is the weight of wet filter cake after filtration, and $W_{2}$ is the weight of filter cake after drying at $105^{\circ} \mathrm{C}$ for $24 \mathrm{~h}$.

The basic characteristics of drilling sludge, including water content, $\mathrm{pH}$ and volatile suspended solids (VSS), were analyzed according to $\mathrm{CJ} / \mathrm{T}$ 221-2005 (China standard for sludge analysis). The sludge viscosity was measured using a rotational viscometer (NDJ-8S, Nirun, China). The supernatant of collected filtrate was filtered through microporous membrane before analysis, and its transmittance was determined using a spectrophotometer (723, Jinghua, China). The morphological character of the conditioned sludge was observed with a microscope (CX41, Olympus, Japan). The sludge surface morphologies of different treated sludge samples were monitored with a scanning electron microscope (S4800, Hitachi, Japan). The test samples were frozen and vacuum dried prior to observation. The zeta potential was tested using Zetasizer Nano ZS90 (Malvern, UK). The particle size distributions of drilling sludge were analyzed by a laser diffraction particle size analyzer (Mastersize 2000, Malvern, UK) after dispersing drilling sludge samples in distilled water.

Table 3 Analysis of variance for the orthogonal experiments

\begin{tabular}{llrr}
\hline Source of variance & Degree of freedom & $\begin{array}{l}\text { Sum of the deviation } \\
\text { square }\end{array}$ & Mean square \\
\hline $\begin{array}{l}\text { Sodium persulfate } \\
\text { concentration }\end{array}$ & 2 & 9.01 & 4.51 \\
Ferrous sulfate concentration & 2 & 6.79 & 305.35 \\
Stirring speed & 2 & 2.79 & 3.39 \\
Reaction time & 2 & 4.69 & 1.40 \\
Pooled error & 9 & 0.13 & 2.35 \\
\end{tabular}




\section{Results and discussion}

\subsection{Effect of sodium persulfate concentration}

The effects of the sodium persulfate concentration on drilling sludge dewaterability are shown in Fig. 1, using sulfate radical oxidation. The water content for blank sample which means no sodium persulfate and ferrous sulfate added in the drilling sludge is $35.04 \%$, and SRF at this time is around $3.29 \times 10^{13} \mathrm{~m}$ $\mathrm{kg}^{-1}$. We can see the drilling sludge dewaterability was improved obviously in the presence of sodium persulfate and ferrous sulfate. The value of both water content and SRF declined when the sodium persulfate dosage increased from 0.8 to $2.4 \%$. The water content decreased from $33.69 \%$ to $30.81 \%$ when the sodium persulfate concentration increased from $0.8 \%$ to $1.6 \%$, and then it keep about $31 \%$ when the sodium persulfate concentration increased from $1.6 \%$ to $2.4 \%$. The SRF also decreased from $1.76 \times 10^{13} \mathrm{~m} \mathrm{~kg}^{-1}$ to $1.13 \times 10^{13} \mathrm{~m} \mathrm{~kg}^{-1}$, it has similar curve shape to the water content. The experimental results were attributed to the generation of sulfate radical (eqn (1)), which degraded organic materials and disintegrated the sludge fiocs. The trapped water was transformed into free water during the process. Sodium persulfate is the source of $\mathrm{Fe}^{2+}$ / persulfate oxidation, so the increased sodium persulfate concentration would produce more radicals. However, the dewaterability would remain unchanged when the sodium persulfate concentration was over $1.6 \%$. This might be due to the insufficiency of ferrous sulfate limited the oxidization process. $^{21}$

Sulfate radical oxidation process promoted the hydrolysis of recalcitrant organic substances in the sludge, resulting in the variation of volatile suspended solids and transmittance of the supernatant. Fig. 2 shows the effect of sodium persulfate concentration on the VSS and transmittance during sludge disintegration. The VSS in the sludge decreased rapidly from $8798 \mathrm{mg} \mathrm{L}^{-1}$ down to $5988 \mathrm{mg} \mathrm{L}^{-1}$ when sodium persulfate concentration increases from $0.8 \%$ to $1.6 \%$. The higher concentration of the treating agent did not achieve more VSS removals when it exceeded $1.6 \%$, this result is consistent with

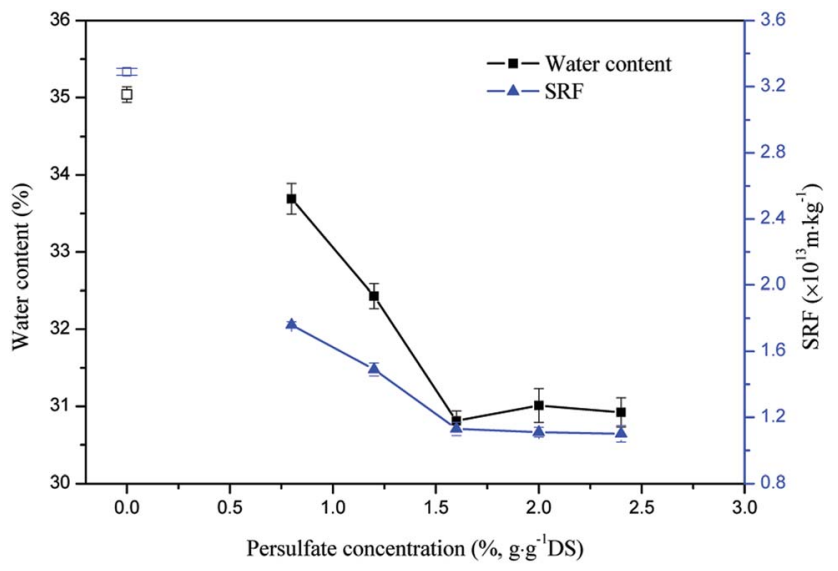

Fig. 1 Effect of sodium persulfate concentration on sludge dewaterability (except the blank sample points, the ferrous sulfate concentration is $0.8 \%)$.

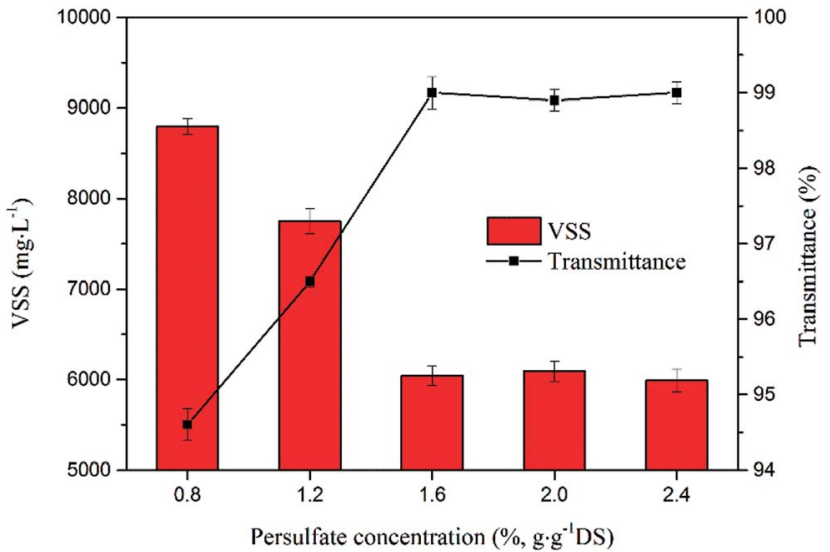

Fig. 2 Effect of sodium persulfate concentration on VSS and transmittance (the ferrous sulfate concentration was $0.8 \%$ ).

Zhen et al. ${ }^{34}$ It implied that the oxidation system accelerated the volatile solids destruction and hydrolysis. The curve of the supernatant transmittance displayed an opposite trend. The increase of transmittance was attributed to the degradation of organic pollutants in the solution.

\subsection{Effect of ferrous sulfate concentration}

Fig. 3 presents the effect of ferrous sulfate concentration on the sludge dewatering. As an initiator, ferrous sulfate had a significant infiuence on the value of water content and SRF. The water content decreased from $34.73 \%$ to $30.81 \%$ with ferrous sulfate dosage rising from 0 to $0.8 \%$, but tended to increase when ferrous sulfate dosage exceeded $0.8 \%$. The lowest water content was achieved at $0.8 \%$. The variation trend of SRF was different from water content. With ferrous sulfate increasing from 0 to $1.6 \%$, the value of SRF declined obviously and tended to stable. The value of SRF reduced from $2.97 \times 10^{13} \mathrm{~m} \mathrm{~kg}^{-1}$ to $1.07 \times$ $10^{13} \mathrm{~m} \mathrm{~kg}^{-1}$. The improvement of dewaterability probably was caused by $\mathrm{Fe}^{2+}$ activated sodium persulfate, and the produce of sulfate radical could change sludge fiocs structure. However, the excess ferrous sulfate deteriorated sludge dewatering by quenching sulfate radical through (eqn (4)). ${ }^{35}$

$$
\mathrm{Fe}^{2+}+\mathrm{SO}_{4}^{-\cdot} \rightarrow \mathrm{Fe}^{3+}+\mathrm{SO}_{4}^{2-}
$$

To further evaluate the influence of ferrous sulfate on sludge characteristic, VSS and transmittance of the supernatant were also analyzed as shown in Fig. 4. The VSS of sludge decreased to a minimum at first and then increased with increasing dosage of ferrous sulfate. Conversely, the transmittance of the supernatant (which optimal transmission wavelength is $660 \mathrm{~nm}$ ) rose rapidly when the ferrous sulfate concentration is lower, but later this tendency was reverse when the ferrous sulfate dosage was added over $0.8 \%$. The phenomenon indicated that there was a threshold concentration of ferrous sulfate during sludge disintegration by sulfate radical oxidation. Therefore, excessive ferrous sulfate could not result in improving the release of the water trapped among organics. 

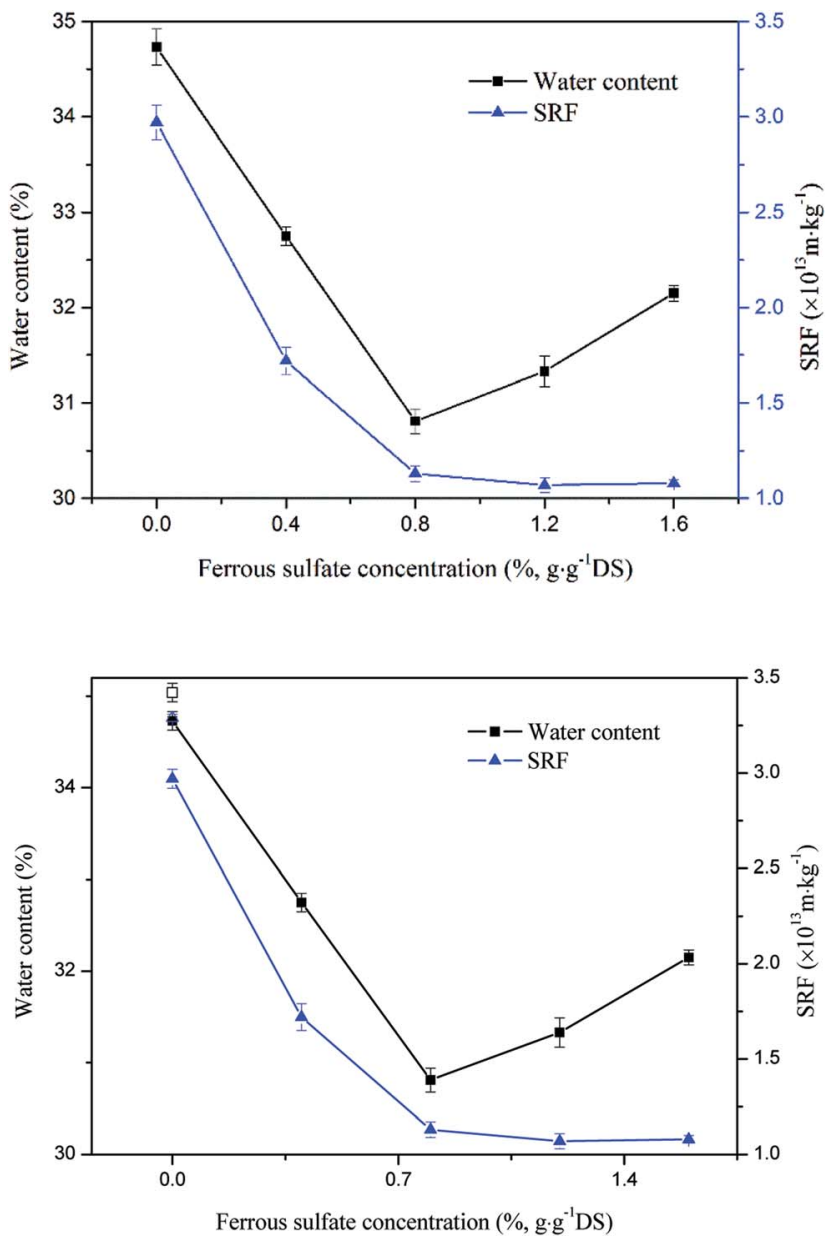

Fig. 3 Effect of ferrous sulfate concentration on sludge dewaterability (except the blank sample points, the sodium persulfate concentration was $1.6 \%)$.

\subsection{Effect of reaction time}

The degree of oxidation of drilling sludge was also decided by reaction time. The effects of reaction time on the sludge dewatering are shown in Fig. 5. The water content and SRF decreased at first and then tended to be stable with reaction

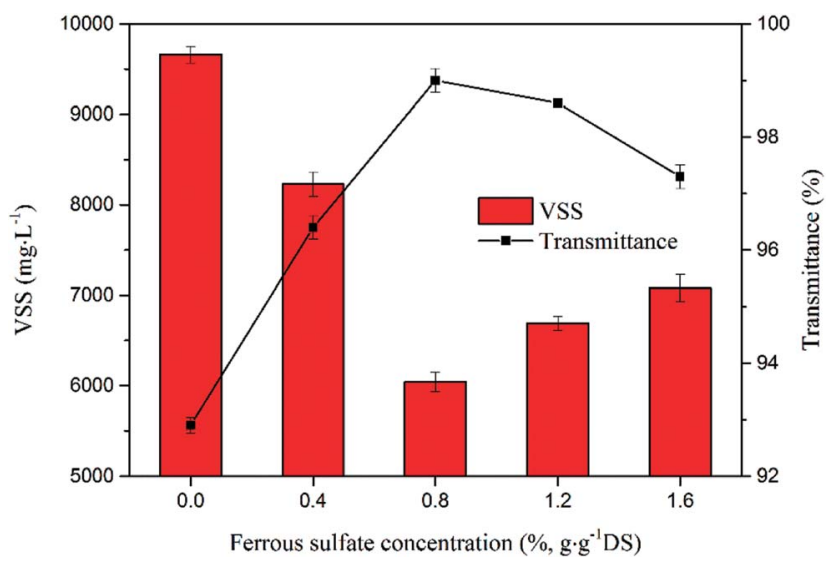

Fig. 4 Effect of ferrous sulfate concentration on VSS and transmittance (the sodium persulfate concentration was $1.6 \%$ ).

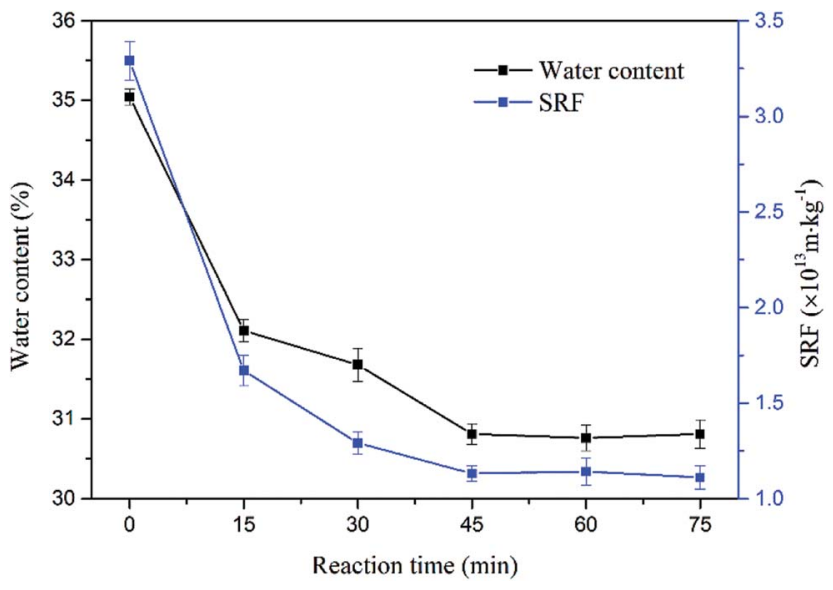

Fig. 5 Effect of reaction time on sludge dewaterability (the sodium persulfate concentration was $1.6 \%$, the ferrous sulfate concentration was $0.8 \%$ and the stirring speed was $300 \mathrm{rpm}$ ).

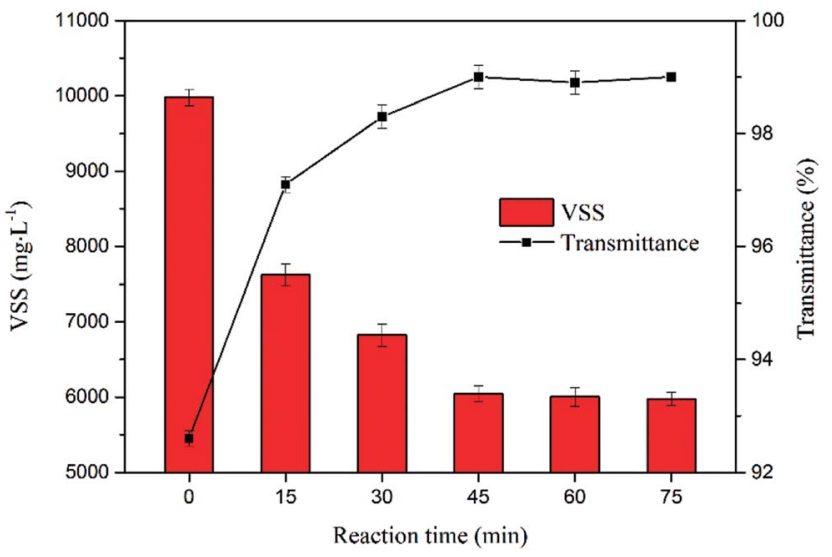

Fig. 6 Effect of reaction time on VSS and transmittance.

time increasing. When the reaction time was $15 \mathrm{~min}$, the reduction of them was larger, and the change was little after the reaction time reached $45 \mathrm{~min}$. It was likely that the reaction rate of sodium persulfate and ferrous sulfate was relatively large in the first $15 \mathrm{~min}$, so there was a significant decrease of water content and SRF. After $45 \mathrm{~min}$, the sulfate radical oxidation had reached equilibrium.

The effect of reaction time on VSS and transmittance of the supernatant is shown in Fig. 6. Within first $15 \mathrm{~min}$, ferrous sulfate and sodium persulfate reacted rapidly to form sulfate radicals, and the organic matter in the sludge was oxidized and degraded. Consequently, the VSS decreased markedly and transmittance of the supernatant increased significantly. After $45 \mathrm{~min}$, there was no obvious change with the extension of reaction time. The results further indicated the oxidation reaction had reached equilibrium.

\subsection{Effects of ultrasound assisted sulfate radical oxidation}

The ultrasound assisted sulfate radical oxidation was performed at ultrasonic power of 0,40 and $80 \mathrm{~W}$ in the time ranged from 0 to $75 \mathrm{~min}$, the concentration of sodium persulfate and 


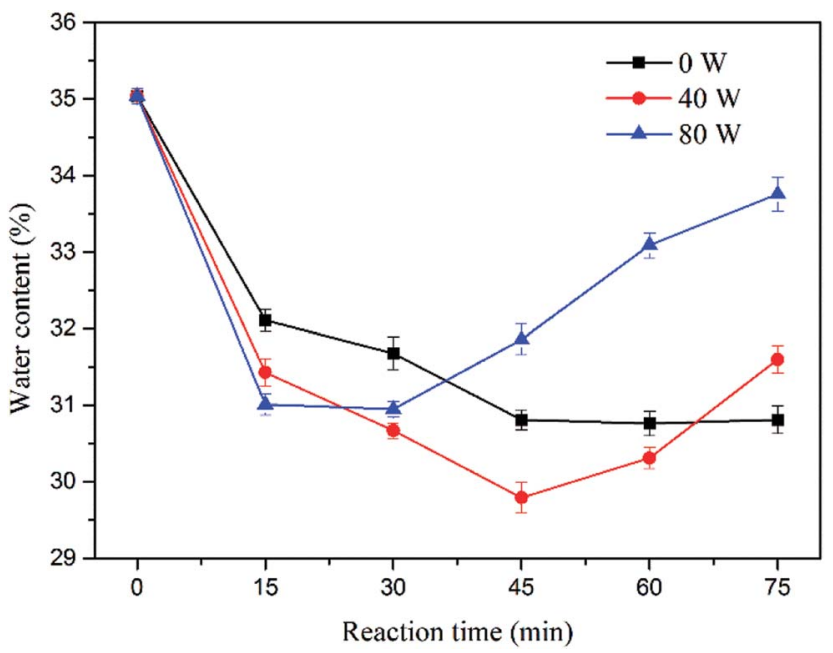

Fig. 7 Effect of ultrasound assisted sulfate radical oxidation on water content.

ferrous sulfate were fixed at $1.6 \%$ and $0.8 \%$, respectively. Fig. 7 and Fig. 8 show the effect of reaction time on sludge dewatering. The water content and SRF had the similar variation trends as the reaction time increased. The value of water content and SRF decreased from $35.04 \%$ and $3.29 \times 10^{13} \mathrm{~m} \mathrm{~kg}^{-1}$ to $30.81 \%$ and $1.11 \times 10^{13} \mathrm{~m} \mathrm{~kg}^{-1}$ with sulfate radical oxidation alone after $75 \mathrm{~min}$. When the reaction time increased from $45 \mathrm{~min}$ to $75 \mathrm{~min}$, the sludge dewaterability kept stable. By contrast, the water content and SRF of sludge declined to $29.79 \%$ and $0.89 \times$ $10^{13} \mathrm{~m} \mathrm{~kg}^{-1}$ when the reaction time reached to $45 \mathrm{~min}$ with $40 \mathrm{~W}$ of ultrasound combination. With the ultrasonic reaction time increasing, both water content and SRF did not further decrease, but instead actually increased. The results might be caused by the excessive increase of supplied ultrasonic energy. To further investigate the impact of energy inputs on the dewaterability, a higher ultrasonic power $(80 \mathrm{~W})$ was applied to the system under the same reaction time. The water content and SRF diminished when the reaction time increased from 0 to

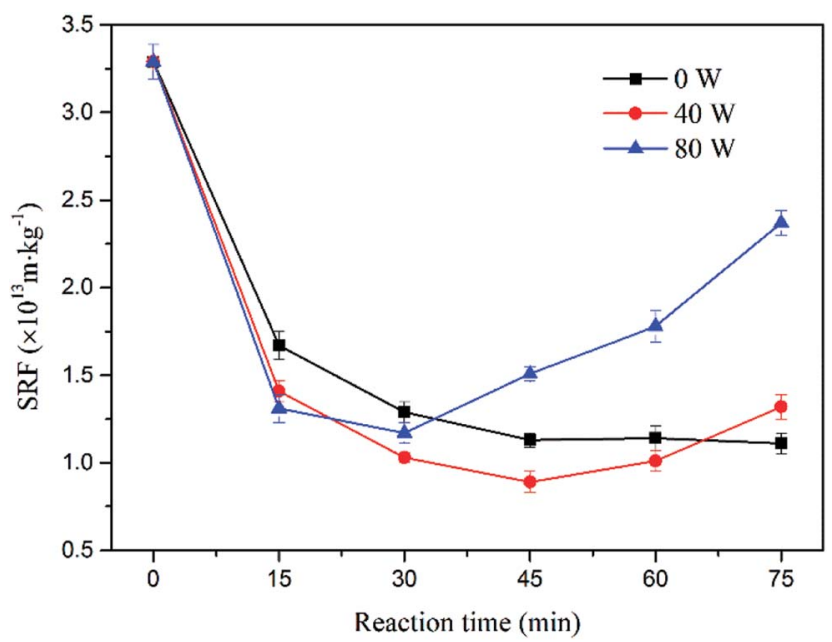

Fig. 8 Effect of ultrasound assisted sulfate radical oxidation on SRF.

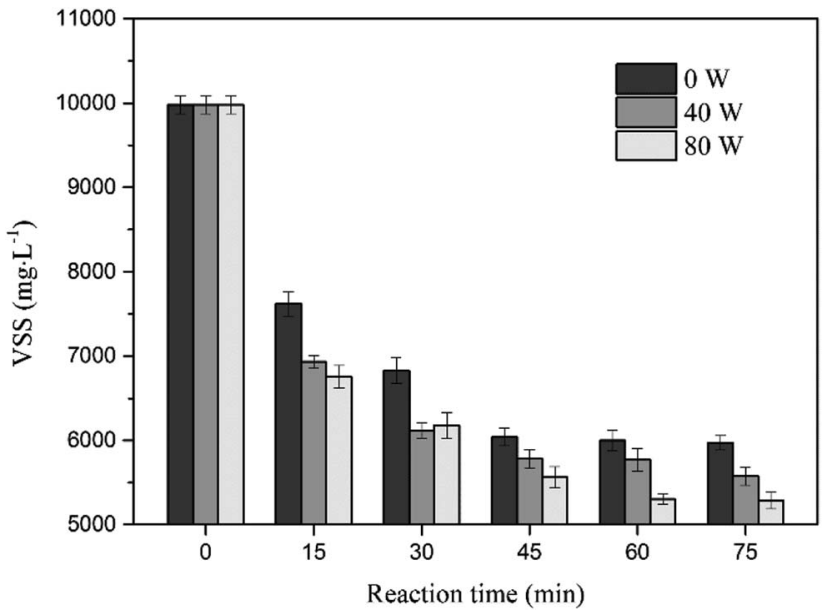

Fig. 9 Effect of ultrasound assisted sulfate radical oxidation on VSS.

$30 \mathrm{~min}$. The optimal values of water content and SRF were $30.95 \%$ and $1.17 \times 10^{13} \mathrm{~m} \mathrm{~kg}^{-1}$ at $30 \mathrm{~min}$. With the extension of the ultrasonic reaction time, the sludge dewaterability began to markedly deteriorate. The results indicated that there was an optimal ultrasonic energy level to improve sludge dewatering and reduce reaction time during the ultrasound assisted sulfate radical oxidation, and the high energy dosage has no beneficial effect on improving of dewaterability. It means the high energy inputs not only accelerate sulfate radical oxidation and disrupt floc structure but also reduce floc size. The small particles provide large surface area for absorbing water, ${ }^{36}$ leading to deterioration of sludge dewaterability.

The influence of ultrasound assisted sulfate radical oxidation on the sludge disintegration in terms of VSS and transmittance of the supernatant are shown in Fig. 9 and 10. The VSS of the sludge decreased with prolonging reaction time and rising ultrasonic energy by both sulfate radical oxidation and ultrasound assisted sulfate radical oxidation. The ultrasound irradiation resulted in more reduction of the VSS concentration. As shown in Fig. 9, the sludge disintegration by both processes was very fast in the first $15 \mathrm{~min}$, and then the velocity decreased with time. The minimum value of VSS was $5973 \mathrm{mg} \mathrm{L}^{-1}$ at 75 min of reaction time without ultrasound treatment, and the reduction of VSS was $40.15 \%$. Whereas under the same condition, the VSS for ultrasound assisted sulfate radical oxidation reached to its minimum of $5290 \mathrm{mg} \mathrm{L}^{-1}$ while the ultrasonic power was $80 \mathrm{~W}$, and the reduction rate of VSS was $46.99 \%$. In these two processes, $\mathrm{Fe}(\mathrm{II})$-activated persulfate oxidation played a major role in sludge disintegration. During the ultrasound assisted sulfate radical oxidation, ultrasound produced hydroxyl radical and provided local extreme conditions, which further promoted the oxidation reaction. The mechanism of ultrasound and sulfate radical oxidation on the decreasing of VSS are different. For ultrasound, high speed microjet were generated to shear the sludge physically so that the structures of flocs can be collapsed. ${ }^{37}$ The organics inside the flocs were thus exposed, broken into smaller volumes and in the meantime, transferred to the solution if soluable such as hydrophilic 


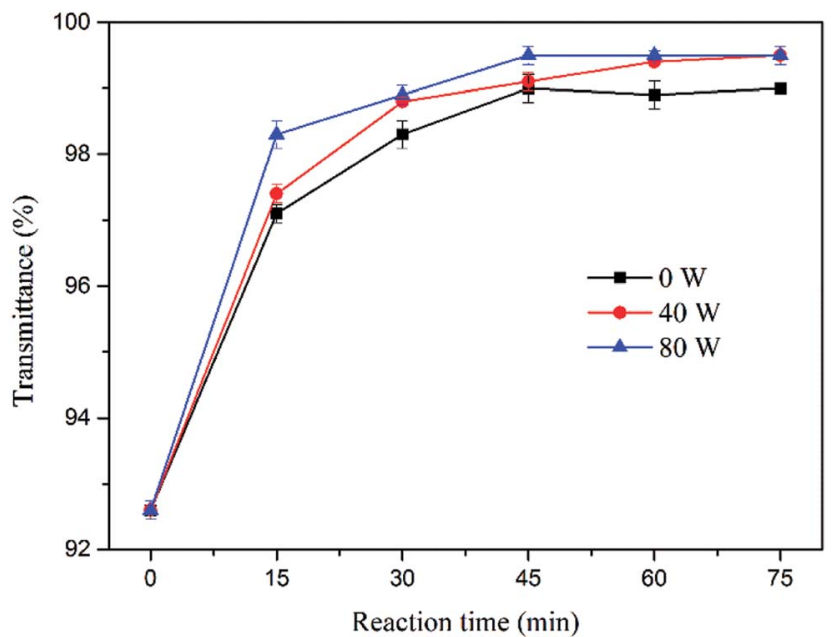

Fig. 10 Effect of ultrasound assisted sulfate radical oxidation on transmittance of the supernatant.

ones. ${ }^{38}$ While during the sulfate radical oxidation process, the organic carbons in sludge floc were decreased due to the chemical oxidation. ${ }^{15}$

Fig. 10 shows the transmittance of the supernatant was obviously increased with the rising reaction time for both pretreatment, and it changed slightly when the reaction time reached to $45 \mathrm{~min}$. Ultrasonic coupling could further increase the transmittance and decrease the time required for equivalent effect by individual sulfate radical oxidation. Dissolved organic matter in the supernatant was further oxidized, resulting in the enhancement of transmittance.

\subsection{Energy consumption}

The optimal condition is $1.6 \%$ sodium persulfate, $0.8 \%$ ferrous sulfate, $40 \mathrm{~W}$ ultrasonic power and 45 min reaction time. The ultrasonic energy consumption is around $0.5 \mathrm{~kW} \mathrm{~h}$ per $\mathrm{kg}$ of removed water and the sodium persulfate and ferrous sulfate with low price for the industrial grade can be reused in the industrial process. We compared with the energy consumption results with other results reported in literatures for different assisted conditioning (or hybrid) methods. ${ }^{39}$ For thermal drying, the energy consumption is around 0.616 to 1.2 $\mathrm{kW} \mathrm{h}$ per $\mathrm{kg}$ of water removed. ${ }^{5}$ Electro-dewatering (EDW) is an alternative emerging and energy-efficient technology that provides improved liquid/solids separations in the dewatering of wastewater sludge. Mahmoud demonstrated that to achieve a dryness fraction of $50 \%$, the optimum EDW process had an energy consumption of $0.30 \mathrm{~kW}$ h per $\mathrm{kg}$ of water removed. ${ }^{28} \mathrm{In}$ this research, we achieve a dryness fraction of $70 \%$ with the optional condition, which ultrasonic energy consumption is around $0.5 \mathrm{~kW} \mathrm{~h}$ per $\mathrm{kg}$ of removed water. Thus, the optional condition in the energy consumption for our research can be acceptable.

\subsection{Variation of sludge properties}

3.6.1. Microstructure of sludge. To intuitively illustrate the possible dewatering mechanisms, floc morphology and surface morphological structure were characterized. Fig. 11 provides optical microscopic observations of raw sludge and conditioned sludge by different pretreatments. The morphology of raw sludge (Fig. 11a) was large and loose floc matrix, which appeared dark brown, and organic polymers bound adjacent particles, causing steric stability of sludge. A part of water would be wrapped in the floc matrix. Fig. 11b presents the sludge treated by ultrasound alone. Ultrasound treatment broke the flocs to a certain extent, but the color of sludge flocs was still dark brown without significant change. The result indicated that there was no obvious effect on sludge oxidation only under the ultrasound conditioning. After the Fe(II)-activated persulfate oxidation (Fig. 11c), sludge floc matrix became dispersed, and most of junction structure between flocs collapsed. Besides, the conditioned sludge exhibited brick-red, because organic matter of sludge was degraded during the oxidation process. The sludge treated with ultrasound assisted sulfate radical oxidation is shown in Fig. 11d. The flocs were further broken into fragments, and the inorganic level of sludge was increased. Compared to the Fig. 11c, ultrasound performed a synergistic impact on the completed disintegration of sludge flocs.

Fig. 12 displays SEM images of typical flocs before and after different pretreatment. The variation of surface morphological structure has close relation to the sludge dewaterability. The raw sludge presented clusters structure with rough floc blocks and flake like surface (Fig. 12a). After the ultrasound conditioning, the surface of flocs became smooth slightly, but failed to show significant impact (Fig. 12b). Fig. 12c shows flake like structures mostly disappeared and were instead of irregular ones by sulfate radical oxidation in the absence of ultrasound. Flake like structures almost entirely faded away after treatment with the combined methods (Fig. 12d). The remaining rigid lattice structure could provide unblocked outflow channels for water release, which was beneficial to improving sludge dewatering.
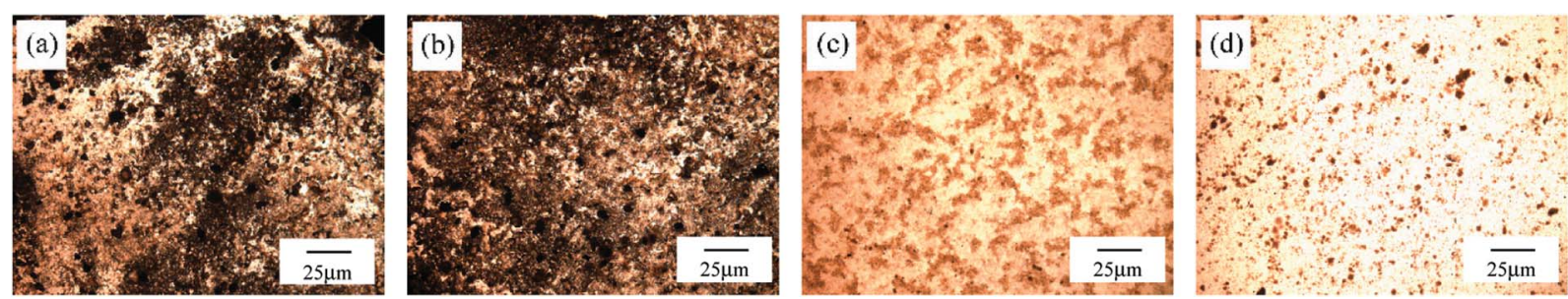

Fig. 11 (a) raw sludge, (b) sludge conditioned with ultrasound for 45 min at 40 W, (c) sludge conditioned with sulfate radical oxidation for 45 min and (d) sludge conditioned by ultrasound assisted sulfate radical oxidation for 45 min at $40 \mathrm{~W}$. 

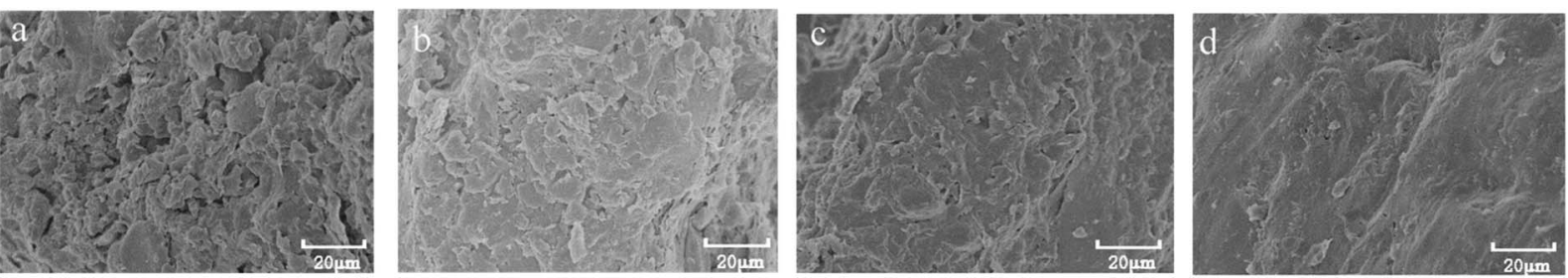

Fig. 12 SEM images of the floc structure of (a) raw sludge, (b) sludge conditioned with ultrasound for 45 min at $40 \mathrm{~W}$, (c) sludge conditioned with sulfate radical oxidation for $45 \mathrm{~min}$ and (d) sludge conditioned by ultrasound assisted sulfate radical oxidation for $45 \mathrm{~min}$ at $40 \mathrm{~W}$.

3.6.2. Variation of particle size distribution and zeta potential. In order to further reveal the disintegration of sludge flocs, the variation of the cumulative particle size distribution of untreated and treated sludge is shown in Fig. 13. The size distribution of raw sludge had a broad range, and the mean particle size was $11.4 \mu \mathrm{m}$. After conditioning under the three different processes, the amount of large flocs all decreased to small ones. In contrast, the particle size of sludge only changed slightly with conditioning of Fe(II)-activated persulfate oxidation, and the mean particle size decreased from $11.4 \mu \mathrm{m}$ to $10.7 \mu \mathrm{m}$. This result was attributed to the two conflicting processes: the oxidization of flocs by the generation of sulfate radical would disintegrated the flocs into smaller particles, while the presence of $\mathrm{Fe}^{2+}$ could neutralize the negative charge, resulting in floc aggregation, which counteracted to the effect of disintegration. In the case of ultrasound treatment, the mean particle size was $4.3 \mu \mathrm{m}$ and decreased by over $62 \%$. The great reduction rate of particle size was caused by ultrasonic cavitation mentioned above. Ultrasound assisted sulfate radical oxidation had a further impact on the decrease of particle size, and the mean particle size was measured at $1.3 \mu \mathrm{m}$. The obvious increase in the number of small particles indicated that ultrasound assisted sulfate radical oxidation could efficiently facilitate the disruption of sludge flocs. As a result, the floc structure transformed into a small and compact form and the bound water was released, leading to the improvement of sludge dewaterability.

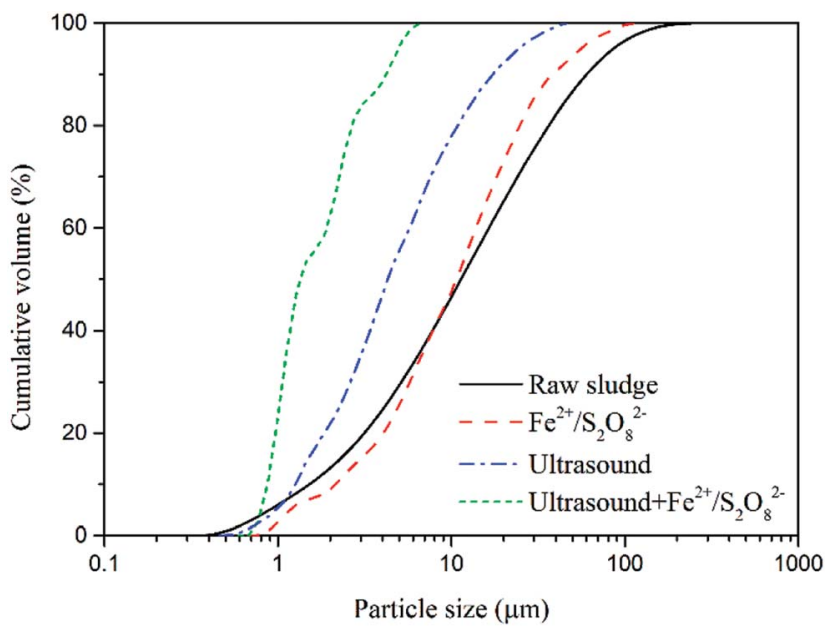

Fig. 13 Particle size distribution of the sludge with different treatments.
Fig. 14 presents the zeta potential of sludge before and after the treatment. The original value of particles zeta potential was $-23.2 \mathrm{mV}$. After the $\mathrm{Fe}(\mathrm{II})$-activated persulfate oxidation treatment, the zeta potential increased sharply to the value of $-0.5 \mathrm{mV}$, since the cations from the reaction system neutralized the negative charge of sludge by providing positive charge. The presence of ultrasound would decrease the zeta potential of sludge. After the treatment with ultrasound alone, the zeta potential declined from $-23.2 \mathrm{mV}$ to $-36.7 \mathrm{mV}$. Similarly, the zeta potential decreased from $-0.5 \mathrm{mV}$ to $-18.5 \mathrm{mV}$ with the combined pretreatment. As ultrasound treatment could disintegrate the flocs and expose more surfaces, and plenty of inaccessible charges in the floc interior would emerge, then a rise of negative charges displayed. ${ }^{40}$ Mohapatra et $a l .{ }^{41}$ also reported that higher negative zeta potential would be observed after the ultrasonic pretreatment.

According to the DLVO theory, lower zeta potential can lead to bigger repulsion in the sludge. ${ }^{42}$ Thus, the sludge colloid system can be more stable, which goes against with the efficiency of precipitation and filtration process. On the other side, higher repulsion will promote the shearing effect of ultrasound pretreatment, which will release the bound water in the sludge. ${ }^{15}$

\subsection{Mechanism of sludge dewatering}

Based on the change of sludge properties mentioned above, and inspired from some researches, ${ }^{\mathbf{4 3 4} 4}$ the mechanism of

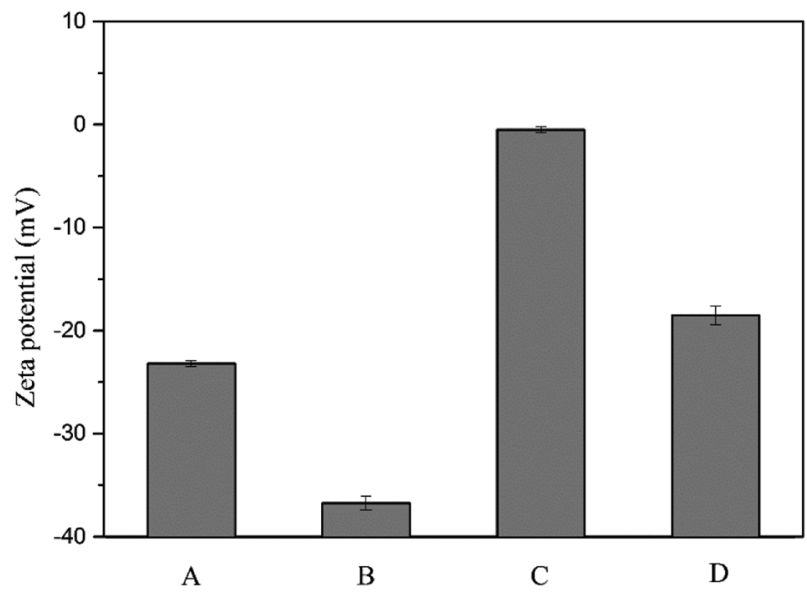

Fig. 14 Zeta potential of the sludge with different treatments. (A) raw sludge, (B) ultrasound, (C) $\mathrm{Fe}^{2+} / \mathrm{S}_{2} \mathrm{O}_{8}{ }^{2-}$ and (D) ultrasound $+\mathrm{Fe}^{2+} /$ $\mathrm{S}_{2} \mathrm{O}_{8}^{2-}$ 


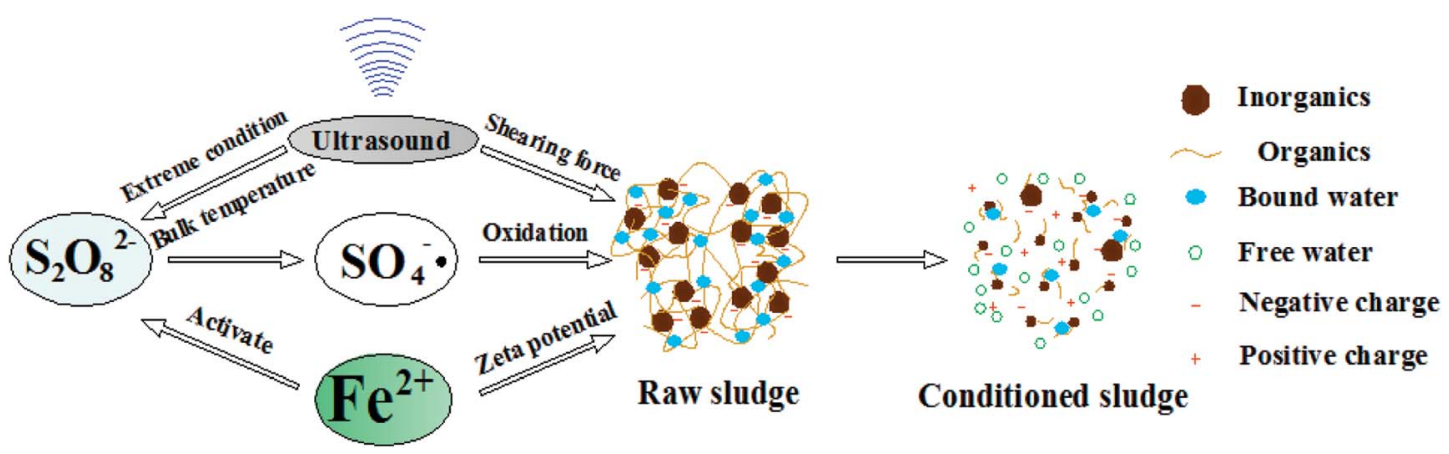

Fig. 15 Mechanism of improving dewatering by ultrasound assisted sulfate radical oxidation. ${ }^{43,44}$

improving dewatering by ultrasound assisted sulfate radical oxidation was depicted in Fig. 15. Since the surrounding liquid is cool, the heat is highly localized to a 'hot-spot' that can reach temperatures and pressures of around $5000 \mathrm{~K}$ and $1000 \mathrm{bar}$, respectively. ${ }^{45}$ Localized high temperatures and pressures from ultrasonically produced cavitation result in homolysis of the $\mathrm{O}-\mathrm{O}$ bond producing sulfate radicals. At the same time, the sound energy can be transformed to heat energy with ultrasound pretreatment, which can promote the activation of sulfate radicals. After that, more sulfate radicals can be generated and the sludge can be collapsed by chemical oxidation. Besides, persulfate sulfate radicals will be activated with ferrous ion and ultrasound, and the sulfate radicals can break the floc chain, releasing the bound water. In addition to the process mentioned above, the presence of $\mathrm{Fe}^{2+}$ neutralizes the negative charge, resulting in floc aggregation, forming a permeable and rigid lattice structure as well as altering the sludge compressibility by remaining porous, ${ }^{\mathbf{4 4}}$ which facilitates the dewatering process and significantly reduces the SRF. Thus, we can conclude that the ultrasound and $\mathrm{Fe}^{2+} / \mathrm{S}_{2} \mathrm{O}_{8}{ }^{2-}$ have synergistic effect on the dewatering process of drilling sludge. After the ultrasound assisted sulfate radical oxidation treatment, the organic components were oxidized and decomposed partially, and bound water was released and transformed to free water. Subsequently, the sludge dewaterability was improved effectively.

\section{Conclusions}

The dewatering of drilling sludge by treatment with ultrasound assisted sulfate radical oxidation was investigated in this research. Furthermore, the physical and chemical properties of the sludge before and after the treatment were examined to analyze the mechanisms. The main conclusions are as follows:

(1) Combining ultrasound with sulfate radical oxidation could further enhance the sludge dewaterability and reduce reaction time compared to individual sulfate radical oxidation method. The treatment with sodium persulfate of $1.6 \%$, ferrous sulfate of $0.8 \%$, ultrasonic power of $40 \mathrm{~W}$ and reaction time of 45 min were determined to be the optimal operating conditions in terms of water content and SRF. In addition, the combined method was able to promote the hydrolysis of organic substances in the sludge, resulting in the decrease of volatile suspended solids and increase of transmittance.

(2) The improvement of sludge dewaterability depended on the disintegration of its flocs, and the cleavage of linkages in polymeric backbone could release the bound water. After ultrasound assisted sulfate radical oxidation, the junctions between flocs collapsed, promoting the trapped water transform to free water. Most of plate like structures disappeared from the surface of flocs, and the remaining rigid lattice structure could form unblocked outflow channels for water release during the filtration. Besides, the disintegration of sludge flocs was confirmed by the decrease of particle size after the treatment. Although organic matter in the sludge was not completely decomposed, ultrasound assisted sulfate radical oxidation could efficiently enhance the dewaterability by the means mentioned above.

\section{Conflicts of interest}

There are no conflicts to declare.

\section{Acknowledgements}

This work is supported by the Natural Science Foundation of China (21606164). The authors are grateful for Jereh Environmental Protection Technology Co., Ltd. for the provision of drilling sludge.

\section{References}

1 P. Yang, T. B. Li, M. H. Wu, X. W. Zhu and X. Q. Sun, Analysis of the effect of polyanionic cellulose on viscosity and filtrate volume in drilling fluid, Mater. Res. Innovations, 2015, 19, 1216.

2 Y. C. Liu, J. Z. Xu and Y. L. Zhang, Environmental Impact and Solidification of Water-based Drilling Fluid, Chin. J. Environ. Eng., 2014, 8, 3417-3422(in Chinese).

3 K. Hu, X. D. Chen, W. X. Huang, B. A. Lu and Y. Chen, Novel Sequential Treatment Methodology for Disposal of WaterBased Waste Drilling Mud, Environ. Eng. Sci., 2012, 29, 669-676. 
4 M. Loginov, M. Citeau, N. Lebovka and E. Vorobiev, Electrodewatering of drilling sludge with liming and electrode heating, Sep. Purif. Technol., 2013, 104, 89-99.

5 A. Mahmoud, J. Olivier, V. Jean, F. Andrew and A. Hoadley, Advances in Mechanical Dewatering of Wastewater Sludge Treatment, Wastewater Reuse and Management, 2012, pp. 253-303.

6 A. Mahmoud, J. Olivier, V. Jean, F. Andrew and A. Hoadley, Electrical field: A historical review of its application and contributions in wastewater sludge dewatering, Water Res., 2010, 44, 2381-2407.

7 A. Mahmoud, A. Fernandez, T.-M. Chituchi and P. Arlabosse, Thermally assisted mechanical dewatering (TAMD) of suspensions of fine particles: Analysis of the influence of the operating conditions using the response surface methodology, Chemosphere, 2008, 72, 1765-1773.

8 J. Xiao, X. Wu, W. Yu, S. Liang, J. Yu, Y. Gu, H. Deng, J. Hu, K. Xiao and J. Yang, Migration and distribution of sodium ions and organic matters during electrodewatering of waste activated sludge at different dosages of sodium sulfate, Chemosphere, 2017, 189, 67-75.

9 G. Feng, W. Tan, N. Zhong and L. Liu, Effects of thermal treatment on physical and expression dewatering characteristics of municipal sludge, Chem. Eng. J., 2014, 247, 223-230.

10 G. Feng, L. Liu and W. Tan, Effect of Thermal Hydrolysis on Rheological Behavior of Municipal Sludge, Ind. Eng. Chem. Res., 2014, 53, 11185-11192.

11 J. Diak, B. Ormeci and C. Proux, Freeze-thaw treatment of RBC sludge from a remote mining exploration facility in subarctic Canada, Water Sci. Technol., 2011, 63, 1309-1313.

12 X. Feng, J. C. Deng, H. Y. Lei, T. Bai, Q. J. Fan and Z. X. Li, Dewaterability of waste activated sludge with ultrasound conditioning, Bioresour. Technol., 2009, 100, 1074-1081.

13 S. H. Guo, G. Li, J. H. Qu and X. L. Liu, Improvement of acidification on dewaterability of oily sludge from flotation, Chem. Eng. J., 2011, 168, 746-751.

14 M. A. Tony, Y. Q. Zhao, J. F. Fu and A. M. Tayeb, Conditioning of aluminium-based water treatment sludge with Fenton's reagent: Effectiveness and optimising study to improve dewaterability, Chemosphere, 2008, 72, 673-677.

15 C. Liu, B. Wu and X. Chen, Sulfate radical-based oxidation for sludge treatment: A review, Chem. Eng. J., 2017, 335, 865-875.

16 J. Vaxelaire, Electro-Dewatering of Anaerobically Digested and Activated Sludges: An Energy Aspect Analysis, Drying Technol., 2014, 32, 1091-1103.

17 S. Sahinkaya, Disintegration of municipal waste activated sludge by simultaneous combination of acid and ultrasonic pretreatment, Process Saf. Environ. Prot., 2015, 93, 201-205.

18 H. Li, Y. Y. Jin, R. B. Mahar, Z. Y. Wang and Y. F. Nie, Effects of ultrasonic disintegration on sludge microbial activity and dewaterability, J. Hazard. Mater., 2009, 161, 1421-1426.

19 S. Na, Y. U. Kim and J. Khim, Physiochemical properties of digested sewage sludge with ultrasonic treatment, Ultrason. Sonochem., 2007, 14, 281-285.
20 C. P. Chu, B. V. Chang, G. S. Liao, D. S. Jean and D. J. Lee, Observations on changes in ultrasonically treated wasteactivated sludge, Water Res., 2001, 35, 1038-1046.

21 D. Q. He, L. F. Wang, H. Jiang and H. Q. Yu, A Fenton-like process for the enhanced activated sludge dewatering, Chem. Eng. J., 2015, 272, 128-134.

22 M. A. Tony, Y. Q. Zhao and A. M. Tayeb, Exploitation of Fenton and Fenton-like reagents as alternative conditioners for alum sludge conditioning, J. Environ. Sci., 2009, 21, 101-105.

23 E. Neyens and J. Baeyens, A review of classic Fenton's peroxidation as an advanced oxidation technique, $J$. Hazard. Mater., 2003, 98, 33-50.

24 G. Y. Zhen, X. Q. Lu, Y. C. Zhao, X. L. Chai and D. J. Niu, Enhanced dewaterability of sewage sludge in the presence of Fe(II)-activated persulfate oxidation, Bioresour. Technol., 2012, 116, 259-265.

25 G. Y. Zhen, X. Q. Lu, Y. Y. Li, Y. C. Zhao, B. Y. Wang, Y. Song, X. L. Chai, D. J. Niu and X. Y. Cao, Novel insights into enhanced dewaterability of waste activated sludge by Fe(II)activated persulfate oxidation, Bioresour. Technol., 2012, 119, 7-14.

26 Y. F. Shi, J. K. Yang, W. B. Yu, S. N. Zhang, S. Liang, J. Song, Q. Xu, N. Ye, S. He, C. Z. Yang and J. P. Hu, Synergetic conditioning of sewage sludge via $\mathrm{Fe} 2+$ /persulfate and skeleton builder: Effect on sludge characteristics and dewaterability, Chem. Eng. J., 2015, 270, 572-581.

27 G. Y. Zhen, X. Q. Lu, B. Y. Wang, Y. C. Zhao, X. L. Chai, D. J. Niu, A. H. Zhao, Y. Y. Li, Y. Song and X. Y. Cao, Synergetic pretreatment of waste activated sludge by Fe(II)activated persulfate oxidation under mild temperature for enhanced dewaterability, Bioresour. Technol., 2012, 124, 2936.

28 M. Citeau, O. Larue and E. Vorobiev, Influence of salt, $\mathrm{pH}$ and polyelectrolyte on the pressure electro-dewatering of sewage sludge, Water Res., 2011, 45, 2167-2180.

29 A. Mahmoud, A. F. A. Hoadley, M. Citeau, J. M. Sorbet, G. Olivier, V. Jean and J. Olivie, A comparative study of electro-dewatering process performance for activated and digested wastewater sludge, Water Res., 2018, 129, 66-82.

30 M. C. Lu, C. J. Lin, C. H. Liao, R. Y. Huang and W. P. Ting, Dewatering of activated sludge by Fenton's reagent, Adv. Environ. Res., 2003, 7, 667-670.

31 J. L. Liang, S. S. Huang, Y. K. Dai, L. Li and S. Y. Sun, Dewaterability of five sewage sludges in Guangzhou conditioned with Fenton's reagent/lime and pilot-scale experiments using ultrahigh pressure filtration system, Water Res., 2015, 84, 243-254.

32 Y. Q. Zheng, L. T. Ke, D. Xia, Y. M. Zheng, Y. P. Wang, H. Li and Q. B. Li, Enhancement of digestates dewaterability by CTAB combined with CFA pretreatment, Sep. Purif. Technol., 2016, 163, 282-289.

33 J. Zou, H. Zhu, F. H. Wang, H. Y. Sui and J. T. Fan, Preparation of a new inorganic-organic composite flocculant used in solid-liquid separation for waste drilling fluid, Chem. Eng. J., 2011, 171, 350-356. 
34 G. Y. Zhen, X. Q. Lu, J. Niu, L. H. Su, X. L. Chai, Y. C. Zhao, Y. Y. Li, Y. Song and D. J. Niu, Inhibitory effects of a shock load of $\mathrm{Fe}(\mathrm{II})$-mediated persulfate oxidation on waste activated sludge anaerobic digestion, Chem. Eng. J., 2013, 233, 274-281.

35 C. H. Yen, K. F. Chen, C. M. Kao, S. H. Liang and T. Y. Chen, Application of persulfate to remediate petroleum hydrocarbon-contaminated soil: Feasibility and comparison with common oxidants, J. Hazard. Mater., 2011, 186, 2097-2102.

36 F. Wang, M. Ji and S. Lu, Influence of ultrasonic disintegration on the dewaterability of waste activated sludge, Environ. Prog., 2006, 25, 257-260.

37 F. Wang, Y. Wang and M. Ji, Mechanisms and kinetics models for ultrasonic waste activated sludge disintegration, J. Hazard. Mater., 2005, 123, 145-150.

38 J. Yang, M. Ji, Y. H. Han, W. H. Liu and X. Q. Zhang, Effect of alkaline and ultrasonic pretreatment on the sludge disintegration, Environ. Sci., 2008, 29, 1002-1007.

39 J. Olivier, A. Mahmoud, V. Jean, J.-B. Conrardy, M. Citeau and E. Vorobiev, Electro-Dewatering of Anaerobically Digested and Activated Sludges: An Energy Aspect Analysis, Drying Technol., 2014, 32, 1091-1103.

40 B. H. Guan, J. Yu, H. L. Fu, M. H. Guo and X. H. Xu, Improvement of activated sludge dewaterability by mild thermal treatment in $\mathrm{CaCl} 2$ solution, Water Res., 2012, 46, 425-432.

41 D. P. Mohapatra, S. K. Brar, R. D. Tyagi and R. Y. Surampalli, Concomitant degradation of bisphenol A during ultrasonication and Fenton oxidation and production of biofertilizer from wastewater sludge, Ultrason. Sonochem., 2011, 18, 1018-1027.

42 J. C. Vogelaar, K. A. De, S. Spijker and G. Lettinga, Bioflocculation of mesophilic and thermophilic activated sludge, Water Res., 2013, 39, 37-46.

43 J. Liu, Q. Yang, D. Wang, X. Li, Y. Zhong, X. Li, Y. Deng, L. Wang, K. Yi and G. Zeng, Enhanced dewaterability of waste activated sludge by Fe(II)-activated peroxymonosulfate oxidation, Bioresour. Technol., 2016, 206, 134-140.

44 H. Z. ChunCai and L. H. XingZhong, Ultrasound enhanced heterogeneous activation of peroxymonosulfate by a bimetallic $\mathrm{Fe}-\mathrm{Co} / \mathrm{SBA}-15$ catalyst for the degradation of Orange II in water, J. Hazard. Mater., 2015, 283, 70-79.

45 R. J. Wood, J. Lee and M. J. Bussemaker, A parametric review of sonochemistry: Control and augmentation of sonochemical activity in aqueous solutions, Ultrason. Sonochem., 2017, 38, 351-370. 